\title{
Image Compression Techniques Using Modified high quality Multi wavelets
}

\author{
M.Ashok \\ Assoc.Professor, \\ S.S.J.EC
}

\author{
Dr.T.BhaskaraReddy \\ Assoc.Professor, \\ S.K.University-ATP
}

\begin{abstract}
- over the past decade, the success of wavelets in solving many different problems has contributed to its unprecedented popularity. For best performance in image compression, wavelet transforms require filters that combine a number of desirable properties, such as orthogonality and symmetry. Advances in wavelet transforms and quantization methods have produced algorithms capable of surpassing the existing image compression standards like the Joint Photographic Experts Group (JPEG) algorithm. For best performance in image compression, wavelet transforms require filters that combine a number of desirable properties, such as orthogonality and symmetry. This paper presents new multi wavelet transform and quantization methods and introduces multi wavelet packets. Extensive experimental results demonstrate that our techniques exhibit performance equal to, or in several cases superior to, the current wavelet filters.
\end{abstract}

Keywords- Multi wavelets; DWT; Image coding; Quantizers; ANN.

\section{INTRODUCTION}

Digital Image Processing is defined as analyzing and manipulating images. Image Compression has become the most recent emerging trend throughout the world. Some of the common advantages image compressions over the internet are reduction in time of webpage uploading and downloading and lesser storage space in terms of bandwidth. Compressed images also make it possible to view more images in a shorter period of time [7].Image compression is essential where images need to be stored, transmitted or viewed quickly and efficiently. The benefits can be classified under two ways as follows: First, even uncompressed raw images can be stored and transmitted easily. Secondly, compression provides better resources for transmission and storage. Image compression is the representation of image in a digitized form with a few bits maintenance only allowing acceptable level of image quality. Compression addresses the problem of reducing the amount of data required to represent a digital image. A good compression scheme is always composed of many compression methods namely wavelet transformation, predicative coding, and vector quantization and so on.

Wavelet transformation is an essential coding technique for both spatial and frequency domains, where it is used to divide the information of an image into approximation and detail sub signals [8]. Artificial Neural Networks (ANN) is also used for image compression. It is a system where many algorithms are used. The ANN is viewed as a graph with various nodes namely source, sink and internal [9]. The input node exists in the input layer and output node exists in the output layer whereas hidden nodes exist in one or more hidden layers.

In ANN various learning method are used namely Unsupervised, Reinforcement learning and Back propagation. Counter Propagation Neural Network (CPN) has become popular since it converges faster. A level of advancement in CPN is forward only Counter Propagation (FOCPN), where correlation based technique is used [10], [11],[12]. Modified forward only Counter Propagation (MFOCPN) is proposed where distance metrics are used to find the winner among the hidden layers neurons [13].

\section{DISCRETE WAVELET TRANSFORM}

Wavelet analysis is based on a decomposition of a signal using an orthonormal family of basic functions [2]. Wavelets are well suited for the analysis of transient and time-varying signals [3]. The wavelet transform has been developed as an alternate approach to Short Time Fourier transform to overcome the resolution problem. The wavelets transform is computed separately for different segments of the timedomain signal at different frequencies. This approach is called Multi resolution analysis (MRA) [4], as it analyzes the signal at different frequencies giving different resolutions. The basic set of wavelets is generated from the mother or basic wavelet is defined as

$$
\psi_{a, b}(t)=\frac{1}{\sqrt{|a|}} \psi\left(\frac{t-b}{a}\right)
$$

Where $a, b \varepsilon \mathrm{R}^{1}$ and $a>0$ The variable ' $a$ ' (inverse of frequency) reflects the scale (width) of a particular basis function such that its large value gives low frequencies and small value gives high frequencies. The variable ' $b$ ' specifies its translation along x-axis in time. The term $1 / a$ is used for normalization [5]. The discrete wavelet transform utilizes two main functions which are scaling and wavelet functions for the multi resolution analysis. The coefficients obtained from scaling and wavelet functions are known as approximate and detail coefficients respectively. The approximate coefficients are low frequency components which are having more significant values than detail coefficients [6]. The scaling and 
wavelet functions are given as follows

$$
\begin{gathered}
W_{\phi}\left(j_{0}, k\right)=\frac{1}{\sqrt{M}} \sum_{x} f(x) \phi_{j_{0}, k}(x) \\
W_{\psi}(j, k)=\frac{1}{\sqrt{M}} \sum_{x} f(x) \psi_{j, k}(x)
\end{gathered}
$$

\section{A. Multi wavelet Transform}

Dyadic wavelet decomposition is achieved by iterative two-channel perfect reconstruction filter bank operations over the low frequency band at each level. This leads to a full tree decomposition as depicted in Figure 1. The final decomposition structure will be a subset of that full tree, chosen by the best basis selection algorithm. Multi wavelets provide one alternative to the wavelet transform. Despite its general success, the wavelet transform often fails to accurately capture high frequency information, especially at low bit rates where such information is lost in quantization noise.

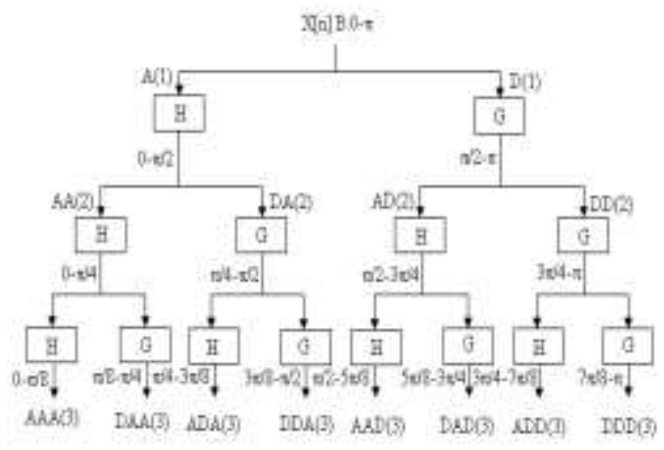

Fig 1: Full tree decomposition for Multi wavelet Transform

A multilevel wavelet filter bank involves iterating the low pass-high pass filtering and down sampling procedure only on the output of the low pass branch of the previous stage. formulated an extension of the octave-band wavelet decomposition to full tree decomposition by allowing the low pass-high pass filtering and down sampling procedure to be iterated also on high pass (band pass) branches in the tree [6].

\section{B. Motivation for Multi wavelets}

Algorithms based on scalar wavelets have been shown to work quite well in image compression. Consequently, there must be some justification to use multi wavelets in place of scalar wavelets. Some reasons for potentially choosing multi wavelets are summarized below [1]: The extra degrees of freedom inherent in multi wavelets can be used to reduce the restrictions on the filter properties.

For example, it is well known that a scalar wavelet cannot simultaneously have both orthogonality and symmetric property. Symmetric filters are necessary for symmetric signal extension, while orthogonality makes the transform easier to design and implement. Also, the support length and vanishing moments are directly linked to the filter length for scalar wavelets. This means longer filter lengths are required to achieve higher order of approximation at the expense of increasing the wavelet's interval of support.
A higher order of approximation is desired for better coding gain, but shorter support is generally preferred to achieve a better localized approximation of the input function. In contrast to the limitations of scalar wavelets, multi wavelets are able to possess the best of all these properties simultaneously. One desirable feature of any transform used in image compression is the amount of energy compaction achieved.

A filter with good energy compaction properties can decorrelate a fairly uniform input signal into a small number of scaling coefficients containing most of the energy and a large number of sparse wavelet coefficients [13]. This becomes important during the quantization since the wavelet coefficients are represented with significantly fewer bits on average than the scaling coefficients. Therefore better performance is obtained when the wavelet coefficients have values clustered about zero with little variance, to avoid as much quantization noise as possible.

\section{ITERATION OF DECOMPOSITION IN MULTI WAVELET TRANSFORM}

Since multi wavelet decompositions produce two low pass sub bands and two high pass sub bands in each dimension, the organization and statistics of multi wavelet sub bands differ from the scalar wavelet case. During a single level of decomposition using scalar wavelet transform, the 2-D image data is replaced with four blocks corresponding to the sub bands representing either low pass or high pass in both dimensions. These sub bands are illustrated in Figure 3-a.

The data in sub band ' $\mathrm{LH}$ ' was obtained from high pass filtering of the rows and then by low pass filtering of the columns. The multi wavelets used here have two channels, so there will be two sets of scaling coefficients and two sets of wavelet coefficients. The multi wavelet decomposition sub bands are shown in Figure 3-b.

For multi wavelets, the $\mathrm{L}$ and $\mathrm{H}$ labels have subscripts denoting the channel to which the data corresponds. For example, the sub band labeled $\mathrm{L} 1 \mathrm{H} 2$ corresponds to data from the second channel high pass filter in the horizontal direction and the first channel low pass in the vertical direction.

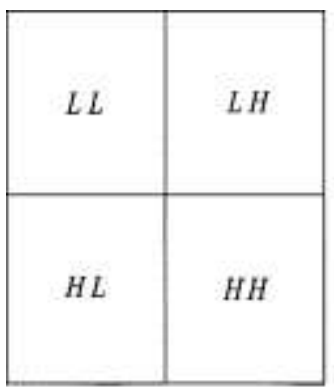

(a)

\begin{tabular}{|l|l|l|l|}
\hline$L_{1} L_{1}$ & $L_{1} L_{2}$ & $L_{1} H_{1}$ & $L_{1} H_{2}$ \\
\hline$L_{2} L_{1}$ & $L_{2} L_{2}$ & $L_{2} H_{1}$ & $L_{2} H_{2}$ \\
\hline$H_{1} L_{1}$ & $H_{1} L_{2}$ & $H_{1} H_{1}$ & $H_{1} H_{2}$ \\
\hline$H_{2} L_{1}$ & $H_{2} L_{2}$ & $H_{2} H_{1}$ & $H_{2} H_{2}$ \\
\hline
\end{tabular}

Fig2.Image sub bands after single-level decomposition for (a) scalar wavelets and (b) multi wavelets. 


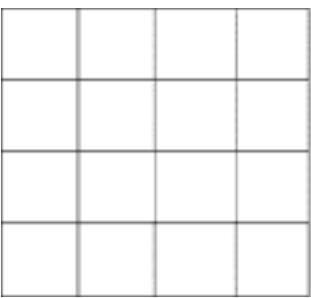

(a)

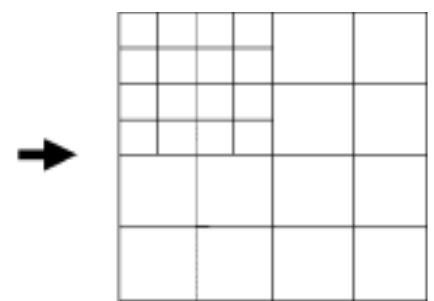

(b)
Fig3. Conventional iteration of multi wavelet decomposition.

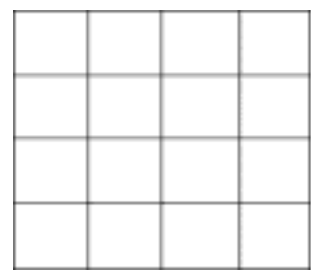

(a)

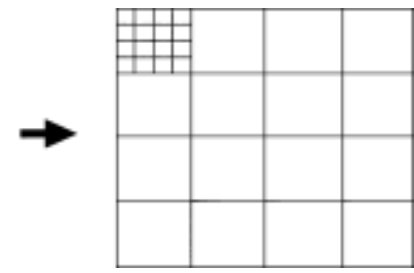

(b)
Fig4. Proposed iteration method for multiwavelet decomposition. Compare to Fig 3.

The multi wavelet decomposition sub bands are shown in Fig. 2(b). For multi wavelets, the labels have subscripts denoting the channel to which the data corresponds. For example, the sub band labeled corresponds to data from the second channel high pass filter in the horizontal direction and the first channel low pass filter in the vertical direction. Scalar wavelet transforms give a single quarter-sized low pass sub band from the original larger sub band, as seen in sub band in Fig. 2(a). In previous multiwavelet literature, multi- level decompositions are performed in the same way. The multiwavelet decompositions iterate on the low pass coefficients from the previous decomposition, [the sub bands in Fig. 2(b)], as shown in Fig. 3. In the case of scalar wavelets, the low pass quarter image is a single sub band. But when the multiwavelet transform is used, the quarter image of "low pass" coefficients is actually a block of sub bands - one low pass and three band pass. This is due to the use of SA multi filters. The scaling function associated with the second channel is band pass since its anti symmetric form gives a zero. Two conclusions may be drawn from these observations. First, since these four sub bands possess different statistical characteristics, mixing them together using the standard multiwavelet decomposition results in subsequent sub bands with mixed data characteristics. This implies that typical quantization schemes that assume the statistics in each sub band are either low pass or high pass will not give the best possible results. Second, since only the sub band is entirely comprised of low pass characteristics, we only need to perform further iterations on that one sub band.

Let $\theta(\mathrm{t})$ and $\varphi(\mathrm{t})$ be the scaling and wavelet functions, respectively, which obey the two-scale equations

$$
\begin{aligned}
& \phi(t)=\sqrt{2} \sum_{k=-\infty}^{\infty} h_{k} \phi(2 t-k), \\
& \psi(t)=\sqrt{2} \sum_{k=-\infty}^{\infty} g_{k} \phi(2 t-k) .
\end{aligned}
$$

Experimental results demonstrate that iterating only on the sub band at each stage in the decomposition does indeed yield better compression than iterating on the four sub bands. Some of these results are depicted in Table I. The 1-2 dB performance improvement indicated in Table $\mathrm{I}$ is typical of this new decomposition scheme. It is also worth noting the computational savings realized on all iterations subsequent to the first: iterating on only the sub band requires one-fourth the numbers of computations as iterating over the four sub bands. The structure of this new improved multi wavelet decomposition method is illustrated in Fig. 4.

\section{A. Quantization: Shuffling}

The quantization method used to generate the results in this paper is the SPIHT zero tree quantizer developed by Said and Pearlman [13]. SPIHT and other zero tree quantizers achieve good performance by exploiting the spatial dependencies of pixels in different sub bands of a scalar wavelet transform. It has been noted [14] that there exists a spatial dependence between pixels in different sub bands in the form of a child-parent relationship. In particular, each pixel in a smaller sub band has four children in the next larger sub band in the form of a block of adjacent pixels. This relationship is illustrated in Fig. 5, which shows three-level scalar wavelet decomposition and some sample pixel relations.

In this figure, each sm all square represents a pixel and each arrow points from a particular parent pixel to its group of children. The importance of the parent-child relation in quantization is this: if the parent coefficient has a small value, then the children will most likely also have small values; conversely, if the parent has a large value, one or more of the children might also. Figure 5 shows the comparison between many multi wavelets when applied for 'Peppers' image at $b_{p p}=1$. From the figure, it is clear that out of all available multi wavelets, $\mathrm{Sa}_{4}$ multi filter performs well. Figure 6 shows a comparison when SPECK compression is applied to wavelet and multi wavelets.

From the figure, it is clear that multiwavelet compression gives $3 \mathrm{~dB}$ improvements against wavelet compression. Figure 7 shows the comparison graph between the algorithms using 'Dabauchies' wavelet. SPECK shows $8 \mathrm{~dB}$ improvements over SPIHT [14].

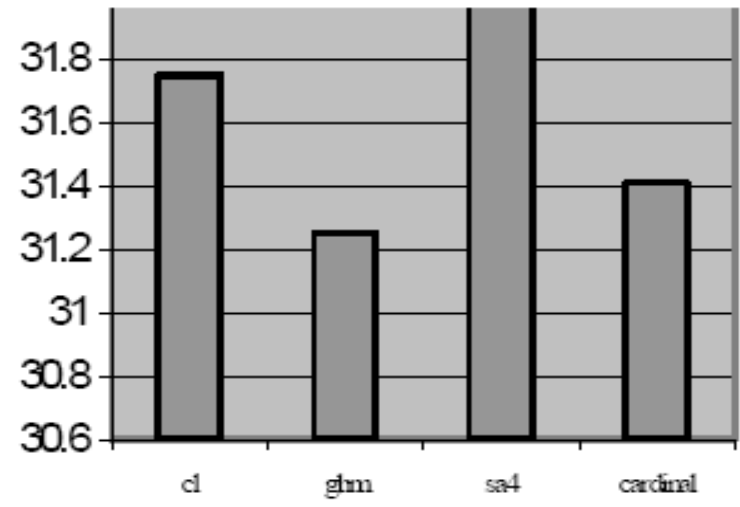

Fig5. PSNR values for various multi wavelets at $\mathrm{CR}=13.33$. 


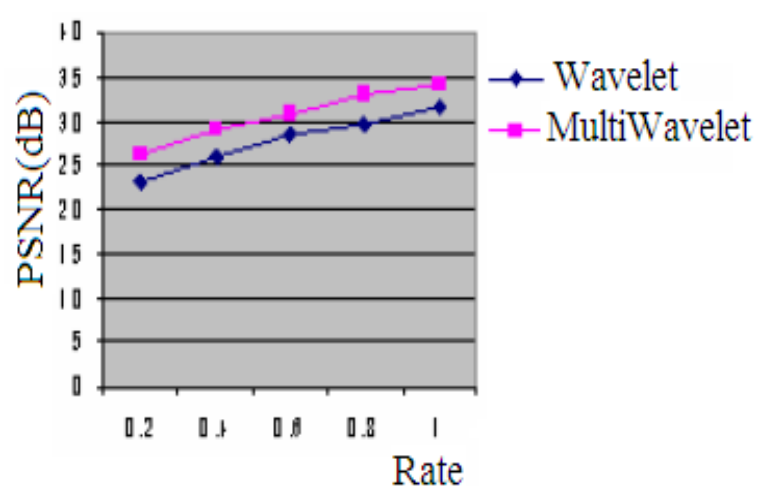

Fig6. Comparison of wavelets vs. multi wavelets

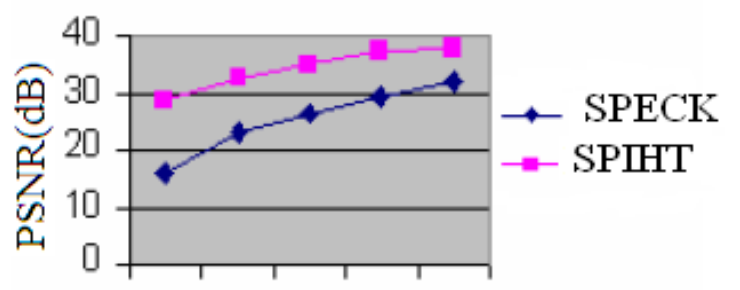

$\begin{array}{lllll}0.2 & 0.4 & 0.6 & 0.8 \quad 1 \text { Rate }\end{array}$

Fig7. Comparison between SPECK and SPIHT

\section{WaVelet Based Fractal Image Coding Algorithm}

\section{A. Encoding Process}

1. Take an image as input of size $512 \times 512$.

2. Calculate $(D W T)$ wavelet up to $i=1,2,3 \ldots \ldots . . N$. levels.

3. For all $N$ levels, divide the $H, V, D$ components of the $i^{\text {th }}$ level in to domain blocks of size $2 B \times 2 B$ and that of $(i+1)^{\text {th }}$ level in to range blocks of size $B \times B$.

4. For domain block, find the best matching range block.

5. Save the mapping information i.e. scaling factor and position of the best matched block into a text file.

6. Also save the nth level approximation component in to the same text file.

7. Transmit this text file as encoded as message.

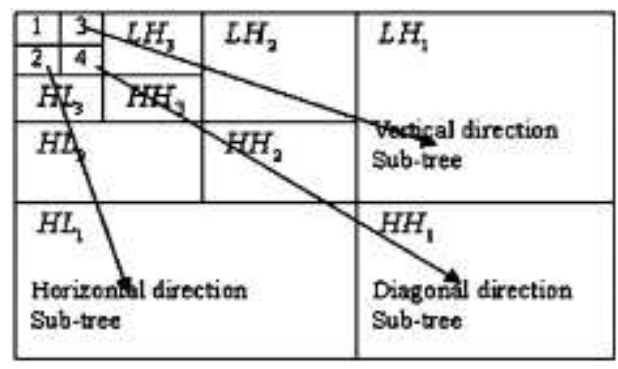

Fig8. Wavelet Sub tree

Fractal Image Compression Technique in Wavelet Domain using Threshold
Encoding Process can be speeded up if a suitable threshold value for MSE is chosen. This speeds up the encoding process because after getting the suitable value it will stop finding the matches for range blocks and domain blocks.

\section{B. Encoding Algorithm}

1. Take an image as input of size $512 \times 512$.

2. Calculate (DWT) wavelet up to $i=1,2,3 \ldots \ldots . . N$. levels.

3. For all $N$ levels, divide the $H, V, D$ components of the $i^{\text {th }}$ level in to domain blocks of size $2 B \times 2 B$ and that of $(i+1)^{\text {th }}$ level in to range blocks of size $B x B$.

4. Choose suitable value for threshold.

5. For every domain block find the matching of range block (depending on threshold value)

6. When the error value is less than the defined threshold value then save the mapping information i.e. scaling factor and position of the best matched block in to a text file.

7. Also save the $N^{\text {th }}$ level approximation component in to the same text file.

8. Transmit this text file encoded as message.

\section{Decoding Process}

1. Read the encoded message file.

2. Take a bitmap image of size $512 \times 512$.

3. For all $N$ levels compute (DWT) wavelet of the image and process the $H, V, D$ components with the help of the data in the encoded message file.

4. Take Inverse Discrete Wavelet Transform for all N levels and get the reconstructed image. Repeat the process for $k$ iterations.

\section{Decoding Algorithm}

The process for decoding the image is the same as that used for wavelet based Fractal Image Compression.

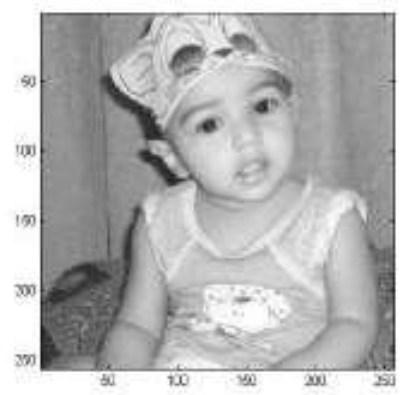

Fig.9: Original Image of baby.tif

Using fractal image compression technique in wavelet domain without threshold. Fig. 5 shows an image lenna.tif of size $512 \times 512$. This image is compressed and decompressed using Fractal Image compression Technique in Wavelet Domain. The encoding time is 124 seconds and the size of the encoded text file is $351 \mathrm{~KB}$. Fig. 6 shows the reconstructed image. 


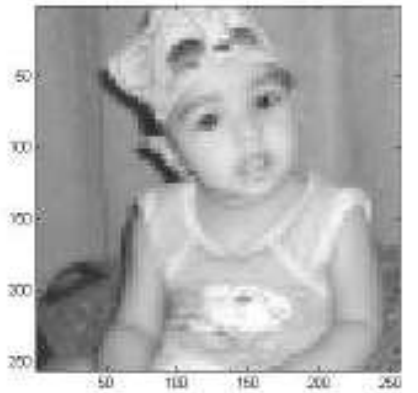

Fig10. Reconstructed image

The same original image Lenna.tif (Fig.6) is compressed and decompressed using Fractal Image

Compression Technique in Wavelet domain with Threshold. The encoding time is 15 seconds and the size of the encoded text file is $344 \mathrm{~KB}$. Fig. 8 shows the reconstructed images. The given tables shows comparison between Fractal Image Compression in Wavelet Domain without Threshold and with Threshold

TABLE 1: SHOWS THE PSNR, DECODING TIME FOR DIFFERENT ITERATION USING FRACTAL IMAGE IN WAVELET DOMAIN WITHOUT THRESHOLD

\begin{tabular}{|l|l|l|}
\hline $\begin{array}{l}\text { Iteration } \\
\text { No }\end{array}$ & PSNR & $\begin{array}{l}\text { Decoding } \\
\text { Time in } \\
\text { sec }\end{array}$ \\
\hline 1 & 20.8637 & 1.9230 \\
\hline 2 & 23.9965 & 2.1236 \\
\hline 3 & 25.5867 & 2.4580 \\
\hline 4 & 29.5050 & 2.7600 \\
\hline 5 & 29.3475 & 2.9830 \\
\hline 6 & 29.3475 & 3.6490 \\
\hline 7 & 29.3475 & 3.7110 \\
\hline 8 & 29.3475 & 4.4480 \\
\hline
\end{tabular}

TABLE 2: PSNR AND DECODING TIME FOR ITERATIONS USING FRACTAL IMAGE COMPRESSION IN WAVELET DOMAIN WITH THRESHOLD

\begin{tabular}{|l|l|l|}
\hline $\begin{array}{l}\text { Iteration } \\
\text { No }\end{array}$ & PSNR & $\begin{array}{l}\text { Decoding } \\
\text { Time in } \\
\text { sec }\end{array}$ \\
\hline 1 & 20.1923 & 1.3240 \\
\hline 2 & 22.6774 & 1.9090 \\
\hline 3 & 23.0321 & 2.1800 \\
\hline 4 & 23.0348 & 2.8700 \\
\hline 5 & 23.0392 & 3.1050 \\
\hline 6 & 23.0398 & 3.6960 \\
\hline 7 & 23.0398 & 3.8570 \\
\hline 8 & 23.0398 & 4.5040 \\
\hline
\end{tabular}

Fig.9 shows comparison of PSNR obtained, using Fractal Image Compression technique in wavelet domain, and wavelet

domain with threshold, for an image Lenna.tif of $512 \times 512$ sizes.

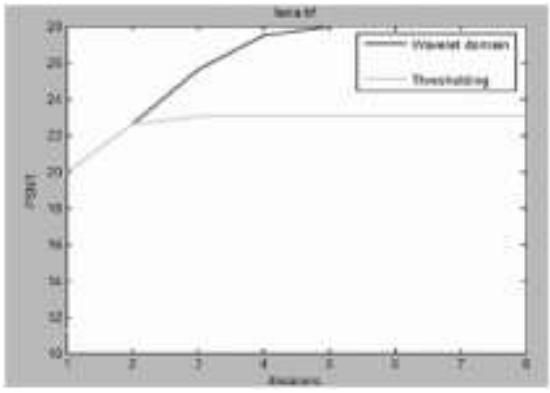

Fig11: Graph Showing Comparison

\section{CONCLUSIONS}

The performance of multi wavelets in general depends on the image characteristics. For the images with mostly low frequency content, (ordinary still images) scalar wavelets generally give better performance. However multi wavelets appear to excel at preserving high frequency content. In particular, multi wavelets better capture the sharp edges and geometric patterns that occur in images. Two new methods for improving the multi wavelet transform have been proposed in this paper: a new multi wavelet decomposition that iterates only on the sub band, and a coefficient shuffling method to improve performance with zero tree-based quantizers. Both methods have been shown to improve the performance of multi wavelet image compression in many cases.

As a result of the collective benefits that JPEG has to offer, despite its computational complexity, it is the lossless compression of choice for mammogram images. JPEG gives way to a mechanism that can provide maximum exchange of mammogram images to healthcare facilitators in an effective, rapid manor, efficient database access and remote access to digital libraries, all aiding to reduce the waiting times for diagnosis.

\section{REFERENCES}

[1] Martin M., "Applications of Multi wavelets to Image Compression," $\mathrm{PhD}$ Thesis, Department of Electrical Engineering, Virginia Polytechnic Institute \& State University, June 1999.

[2] Amara Graps. An introduction to wavelets, IEEE computational science and engineering, summer 1995, Vol 2, No.2.

[3] Ingrid Daubechies, Ten Lectures on Wavelets, SIAM, Philadelphia PA, 1st Edition, 1992.

[4] Amir Averbuch, Danny Lazar, and MosheIsraeli, Image Compression Using Wavelet Transform and Multi resolution Decomposition, IEEE transactions on Image processing, Vol.5, No.1, Jan 1996.

[5] Raghuveer M.Rao, Ajit S. Bopadikar., "Wavelet Transforms", Addison Wesley Longman, Singapore.

[6] Rafel C. Gonzalez, Richard E. Woods., Digital Image Processing, 2nd Edition, Pearson Education.

[7] David Salomon, Data Compression, Springer- Verlag NewYork 3rdEdition, 2005.

[8] Sonja Grgic, Kresimir Kers, and Mislav Grgic, "Image compression using wavelets," ISIE'99- Bled, Slovenia, pp. 99-104.

[9] Hornik K., "Multilayer feed forward networks are universal approximators," Neural Networks, vo1. pp. 359- 366, 1989.

[10] Hecht-Nielsen, "Counterpropagation Networks," Applied Optics, vol.26, pp. 4979- 4984, 1987. 
[11] J.A. Freeman, and D.M. Skapura, Neural Networks, Addison WesleyLongman, 1999.

[12] Donald Woods, "Back and counter propagation aberrations," Proc. IEEE, Neural Networks, vol.1, Pp 473-479,1988.

[13] S.Malarvizhi,U.S.Ragupathy, A.Tamilarasi, "Mammogram Image Compression Using Multiwavelet Transform", Conference Proceedings RTCSP'09

[14] Sudhakar Radhakrishnan and Jayaraman Subramaniam, "Novel Image Compression Using Multi wavelets with SPECK Algorithm" IAJIT,Vol.5,No.1,January2008,pp45-51.

\section{AUTHORS PROFILE}

${ }^{1}$ M.Ashok, Associate Professor, Dept. of Computer Science \& Engg in S. S. J. Engg College, V.N. Palli, Gandipet, A.P, India. His B.Tech from

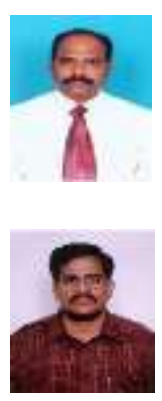

S.K.University and M.Tech from JNTU-Ananthapur. He has over 13 years teaching experience and he has published many papers in National, International conferences and journals. His area of interest is in Image Processing and Embedded Systems.

E-Mail: maram_ashokssjec@yahoo.com

${ }^{2}$ Dr.T.Bhaskara Reddy, Associate Professor Dept. of Computer Science \& Tech in S. K. University, Anantapur, A.P. India.His M.Sc from S.K.University and M.Tech from ANU-Guntur, A.P . He has over 20 years teaching experience and he has published many papers in National, International conferences and journals. His area of interest is in Image Processing and EmbeddedSystems.

E-Mail:bhaskarreddy_sku@yahoo.co.in 